\title{
Correction to: Substituted phenyl containing 1,3,4-oxadiazole-2-yl- but-2-enamides: synthesis and preliminary evaluation as promising anticonvulsants
}

\author{
Nadeem Siddiqui ${ }^{1} \cdot$ Md. Jawaid Akhtar ${ }^{1} \cdot$ M. Shahar Yar $^{1} \cdot$ Priya Ahuja $^{1} \cdot$ Waquar Ahsan $^{2} \cdot$ Sharique Ahmed $^{3}$
}

Published online: 5 April 2019

(c) Springer Science+Business Media, LLC, part of Springer Nature 2019

\section{Correction to:}

Medicinal Chemistry Research (2014) 23:4915-4925

https://doi.org/10.1007/s00044-014-1060-7

The original version of this article unfortunately contained a mistake in university name of Sharique Ahmed.
The correct complete affiliation is Allied Health Department, College of Health Science, University of Bahrain, Kingdom of Bahrain.

The original article can be found online at https://doi.org/10.1007/ s00044-014-1060-7

Nadeem Siddiqui

nadeems_03@yahoo.co.in

$\triangle$ Md. Jawaid Akhtar

jawaid.pharmacist@gmail.com

1 Department of Pharmaceutical Chemistry, Faculty of Pharmacy, Hamdard University, Hamdard Nagar, New Delhi 110062, India

2 Department of Pharmaceutical Chemistry, College of Pharmacy, Jazan University, P. Box No. 114, Jazan, Saudi Arabia

3 Allied Health Department, College of Health Science, University of Bahrain, Manama, Bahrain 